\title{
First detection of the melanoma-predisposing proline-48-threonine mutation of p16 in Hungarians: was there a common founder either in Italy or in Hungary? Márta Széll ${ }^{a, *}$, Klára Balogh ${ }^{\mathrm{b}, *}$, Attila Dobozy ${ }^{\mathrm{a}, \mathrm{b}}$, Lajos Kemény ${ }^{\mathrm{a}, \mathrm{b}}$ and Judit Oláh ${ }^{\mathrm{b}}$
}

The P48T germ line mutation of p16 was detected in a Hungarian multiple primary melanoma patient (deceased at the age of 39 ) with no affected family members. Genetic analysis of the patient and his family revealed that the patient was homozygous for the mutation, whereas his parents (father currently aged 69 and mother 63), who are free from any malignancies and atypical moles, are both heterozygous for the mutation. Our data suggest that the P48T mutation of p16 is a strong melanoma-predisposing factor, but the fact that the heterozygous mutant parents have not yet exhibited melanoma or atypical moles indicates that the penetrance of this allele might depend on modifying factors. The rare P48T germ line mutation of p16 has been reported previously in only four independent studies, all in patients with Italian ancestry. Here, we first report the inheritance of the rare $\mathrm{P} 48 \mathrm{~T}$ mutation of CDKN2A in a Hungarian family with a homozygous multiple primary melanoma member and unaffected heterozygous family members. The question of whether the mutation

\section{Introduction}

The incidence of cutaneous malignant melanoma is increasing worldwide and familial cutaneous melanoma accounts for approximately $10 \%$ of melanoma cases [1]. It is well known from dermatological practice that familial melanoma frequently presents with multiple primary melanomas and clinically atypical moles, and is diagnosed at a much younger age than sporadic cutaneous melanoma [2]. Genetic linkage analysis in large melanoma kindreds identified a melanoma susceptibility locus on human chromosome 9p21 [3], germ line mutations of the cyclindependent kinase inhibitor 2a (CDKN2A/p16INK4A) gene within this chromosomal region are responsible for melanoma susceptibility [4,5]. Mutations of CDKN2A are listed in the Human Gene Mutation Database, http:// wwr.hgmd.cf.ac.uk (accessed 15 August 2006). Some of the listed mutations, detected worldwide, have been implicated in melanoma susceptibility and their inheritance has been intensively studied in the affected families [6], whereas some CDKN2A mutations have been detected only sporadically, and their association with melanoma susceptibility has not been demonstrated in distant ethnic groups. This applies to the P48T mutation of CDKN2A which has so far been detected in only one Italian pancreas carcinoma patient [7], one Italian [8] and detected in Hungary is the result of an independent event, or migration of the founder mutation occurred at some time in the past, necessitates further investigations. Melanoma Res 17:251-254 @ 2007 Lippincott Williams \& Wilkins.

Melanoma Research 2007, 17:251-254

Keywords: CDKN2A gene, germ line mutation, multiple primary melanoma, p16 protein

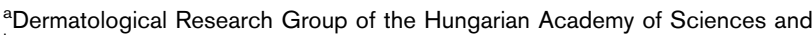
${ }^{b}$ Department of Dermatology and Allergology, University of Szeged, Hungary

Correspondence to Dr Márta Széll, PhD, Dermatological Research Group of the Hungarian Academy of Sciences, University of Szeged, H-6720 Szeged, Korányi fasor 6 , Hungary

Tel: + 3662 545278; fax: + 3662545954 ;

e-mail: szell@mail.derma.szote.u-szeged.hu

*Márta Széll and Klára Balogh have contributed equally to this work.

Received 13 October 2006 Accepted 24 March 2007

one Brazilian [9] melanoma patient, and has been most extensively studied in a large Italian melanoma-prone family [10]. Here, we report the inheritance of the rare P48T mutation of CDKN2A in a Hungarian family with a homozygous multiple primary melanoma member and unaffected heterozygous family members.

\section{Patients and methods}

A 30-year-old Caucasian male presented at our department in 1997 with a thick ulcerated nodular melanoma (p'T3b) in the right femoral region (Fig. 1a). He also suffered from a sporadic form of multiple atypical mole syndrome (Fig. 1b and c). At the time of diagnosis of the primary tumor in the right femoral region which was already $2 \mathrm{~cm}$ in size and bleeding, two early primary melanomas were also diagnosed on his trunk (indicated by arrows in Fig. 1b and c). After removal of these three primary melanomas, he underwent delayed elective radical node dissection from his right groin. The lymph node metastases indicated interferon $\alpha 2 b$ therapy, and he was treated with 10 million units $/ 3$ tw interferon $\alpha 2 b$ for 3 months. Leucopenia and thrombocytopenia complicated the therapy and the patient refused to continue it. During the 8-year follow-up period, a further five new 
Fig. 1
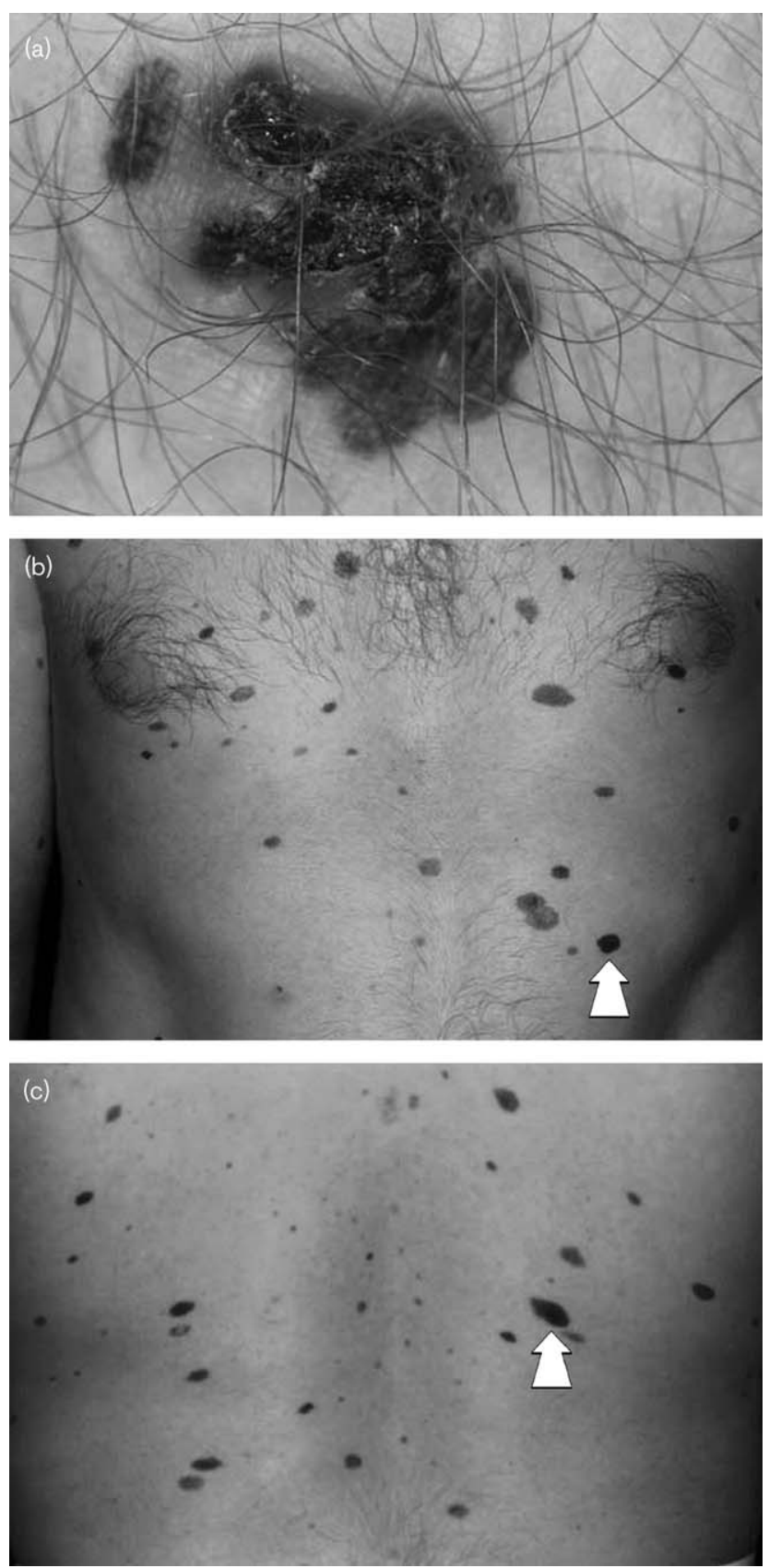

Clinical presentation of the patient. (a) The thick ulcerated nodular melanoma (pT3b) in the right femoral region of the patient at the time of presentation. ( $b$ and $c$ ) The atypical mole syndrome of the patient is apparent on his trunk; the arrows indicate two early primary melanomas diagnosed at the same time as the thick ulcerated nodular melanoma.

early melanomas developed. No internal organ involvement was found for 7 years. In 2004, intra-abdominal lymph node metastases were diagnosed. The patient received bleomycin, vincristine, lomustine and dacarbazine (BOLD) polychemotherapy, which resulted in a slight regression of the metastatic lump. At the end of
2004, radiation therapy was started for the chemoresistant tumor mass in the iliacal and retroperitoneal regions. After irradiation, his condition remained satisfactory for 3 months, with regression of the metastatic lymph nodes. In July 2005, however, intrahepatic metastases were diagnosed. At the end of 2005, he died from multiple symptomatic liver, lung and intracranial metastases.

The appearance of multiple primary melanoma at a relatively young age and the existence of numerous atypical moles suggested a genetic background in this patient, and we therefore decided to investigate the possible involvement of CDKN2A mutations in the pathogenesis. The genetic analysis of the patient and consequently his family members was approved by the local ethics committee. Two millilitres of venous blood was taken, genomic DNA was isolated by using the Genomic DNA Purification Kit of Gentra (Minneapolis, Minnesota, USA), and exons $1 \alpha, 1 \beta, 2$ and 3 of the CDKN2A gene were amplified under the previously reported conditions [11]. The PCR products were purified with the Quantum Prep PCR Kleen Spin Columns of Bio-Rad (Hercules, California, USA). Sequence analysis revealed a homozygote $142 \mathrm{C}>\mathrm{A}$ transversion, which translates the P48T. The mutation is detected in exon $\alpha$, meaning that it affects the fifth amino acid of the second ankyrin repeat of $\mathrm{p} 16$ protein, but the protein sequence of ARF is unaffected.

After detecting this rare mutation in a homozygous form in our patient, we conducted genetic analysis of his parents, his 6-year-old daughter and his wife (Fig. 2a). The father and the mother, currently aged 69 and 63, had no history of any malignant diseases and both are free of any atypical moles. They had no knowledge of consanguinity or the occurrence of familial melanoma among their relatives. Oral sputum samples were taken from the family members and genomic DNA was isolated from the samples with the MagNA Pure Compact system (Roche, Mannheim, Germany). Exon $1 \alpha$ was sequenced with the Resequencing Amplicon probe system (http:// www.ncbi.nlm.nih.gov/genome/probe/reports/probereport), probe ID: RSA001284450.

\section{Results and discussion}

As demonstrated in Fig. 2b, the patient's father, mother and daughter are all heterozygote carriers of the $142 \mathrm{C}>\mathrm{A}$ mutation, and the wife of the patient harbors the wildtype allele. Our results suggest that the patient had inherited one mutant allele from his father and one from his mother, and further transmitted the mutant allele to his daughter.

Germ line mutations in the CDKN2A gene have now been identified in many hereditary melanoma cases, and are associated with the appearance of multiple primary 
Fig. 2

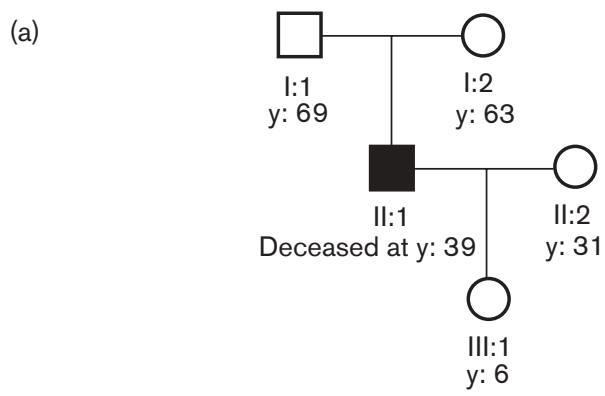

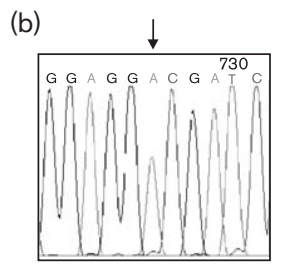

II:1 Homozygote mutant

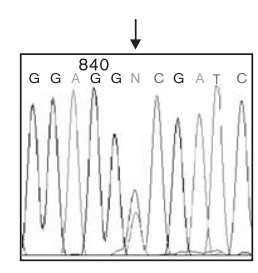

$\mathrm{I}: 1 \quad \mathrm{I}: 2 \quad \mathrm{III}: 1$

Heterozygote mutant

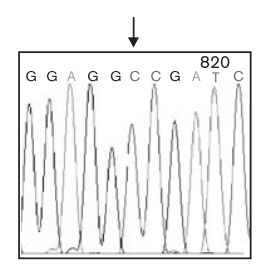

II:2 Wild type (c)

$\begin{array}{ccc}47 & 48 & 49 \\ \text { Arg } & \text { Pro } & \text { lle } \\ \text { AGG } & \text { CCG } & \text { ATC } \\ & \downarrow & \\ & \text { ACG } & \\ & \text { Thr } & \end{array}$

Molecular analysis of the 142C/A CDKN2A variant. (a) The patient (proband) is individual II:1. His father (individual I:1) and mother (individual $\mathrm{l}: 2$ ) are free from malignancies and atypical nevi. (b) Chromatograms showing the $142 \mathrm{C} / \mathrm{A}$ variants; the individuals in the patient's family are listed below the chromatograms according to their genotype. (c) The C/A transversion at nt 142 leads to the Pro/Thr amino acid change in codon position 48 of the p16 transcript.

melanomas $[4,5]$. The gene is composed of four coding regions: exons $1 \alpha, 1 \beta, 2$ and 3 ; exons $1 \alpha, 2$ and 3 code for p16 protein, whereas exons $1 \beta, 2$ and 3 code for ARF protein, both inevitable regulators of the cell cycle $[12,13]$. Various types of mutations are detected on CDKN2A, and their association with different malignant diseases has been documented. Most of the mutations (67 of the 106 so far registered in HGMD; http:// www.hgmd.cf.ac.uk) are missense or nonsense mutations, and their pathogenic role in familial and/or multiple melanoma has been unquestionably demonstrated in distant ethnic populations. For a few missense and nonsense CDKN2A mutations [7,14-16], however, the association with melanoma and/or other malignant diseases has so far been shown only in certain ethnic groups. This is the situation with the transition $142 \mathrm{C}>\mathrm{A}$, which causes the amino acid change $\mathrm{P} / \mathrm{T}$ in codon position 48 (Fig. 2c). This rare CDKN2A germ line mutation has been reported to date in only four cases. It was first detected in an Italian pancreatic cancer patient [7], then in an Italian multiple primary melanoma patient
[8], and subsequently in a Brazilian familial melanoma patient with Italian ancestors [9]: all three patients were heterozygous for the P48T mutation. None of the above studies reported genetic studies on family members, and the possible association of the CDKN2A P48T mutation and the disease phenotype in those cases can therefore not be discussed. The extensive genetic analysis carried out by Della Torre $e t$ al. [10] on a large melanoma-prone Italian family revealed that the heterozygous carriers of the P48T mutation were susceptible not only to melanoma, but also to other forms of malignancies. Similarly, the disease association of the CDKN2A mutation resulting in the $\mathrm{P} 48 \mathrm{~L}$ amino acid change at the same codon was demonstrated beyond question [17]. The associated family analysis clearly revealed the association of this mutation with a very high susceptibility to various types of cancers, including melanoma.

The present case suggests that the CDKN2A P48T mutation is highly associated with the appearance of primary multiple melanoma. Our case lends further support to the multifactorial nature of melanoma predisposition, where the penetrance of a certain allele might depend on modifying factors and can be extremely variable across geographic areas [18], because the heterozygote mutant parents of the patient, at the ages of 69 and 63, are so far free of any malignancies or any atypical nevi, in spite of the fact that both have had a rural lifestyle, with extensive exposure to sunlight. Furthermore, it is extremely rare to discover individuals homozygous for CDKN2A mutations; these cases therefore offer an opportunity to study the effects of potential human null mutations of this ultimate cell cycle regulatory protein. By studying an endogenous Dutch family with a strong founder effect, Gruis et al. [19] observed that homozygous carriers of a small deletion in exon 2 of CDKN2A did not appear to be more seriously affected than heterozygous carriers. Taken together, these results and our present data suggest that CDKN2A mutations in either the heterozygous or the homozygous state are strong susceptibility factors for various malignant diseases, but other additional factors, such as genetic variations of the melanocortin 1 receptor gene in the case of melanoma, are also needed for manifestation of the malignancies.

Although the patient's parents were not aware of consanguinity in their families, the fact that they both harbor the rare $142 \mathrm{C}>\mathrm{A}$ CDKN2A germ line mutation suggests that there must have been such an event or events a few generations previously. It is interesting that the only four previous reports of this mutation involved Italian patients or a Brazilian patient with Italian ancestors. The members of the present Hungarian family are unaware of any Italian family relatives. The question of whether this mutation is the result of an independent event, or whether the founder mutation migrated some 
generations ago from Italy to Hungary, or from Hungary to Northern Italy, demands further examination.

In conclusion, the case we have presented here has confirmed that the rare P48T mutation of p16 is a melanoma-predisposing factor, but our genetic analysis suggests that the heterozygous status itself is not causative of malignant disease: other modifying factors may be needed for the manifestation of these disorders.

\section{Acknowledgements}

The authors thank Mónika Kohajda for her technical assistance. This work was funded by the Grants OTKA NI 62007, GVOP-3.2.1-2004-04-0372/3.0 and ETT531/2006, 500/2006. M.S. is the holder of Bolyai János Research Grant.

\section{References}

1 Hayward NK. Genetics of melanoma predisposition. Oncogene 2003; 22:3053-3062

2 Aitken JF, Green AC, MacLennan R, Youl P, Martin NG. The Queensland Familial Melanoma Project: study design and characteristics of participants. Melanoma Res 1996; 6:155-165.

3 Cannon-Albright LA, Goldgar DE, Meyer L, Lewis CM, Anderson DE, Fountain JW, et al. Assignment of a locus for familial melanoma, MLM, to chromosome 9p13-p22. Science 1992; 258:1148-1152.

4 Sharpless E, Chin L. The INK4a/ARF locus and melanoma. Oncogene 2003; 22: 3092-3098.

5 Czajkowski R, Placek W, Drewa G, Czajkowska A, Uchanska G. FAMMM syndrome: pathogenesis and management. Dermatol Surg 2004; 30: 291-296.

6 Eliason MJ, Larson AA, Florell SR, Zone JJ, Cannon-Albright LA, Samlowski WE, Leachman SA. Population-based prevalence of CDKN2A mutations in Utah melanoma families. J Invest Dermatol 2006; 126:660-666.

7 Moore PS, Zamboni G, Falconi M, Bassi C, Scarpa A. A novel germline mutation, $\mathrm{P} 48 \mathrm{~T}$, in the CDKN2A/p16 gene in a patient with pancreatic carcinoma. Hum Mutat 2000; 16:447-448.
8 Mantelli M, Pastorino L, Ghiorzo P, Barile M, Bruno W, Gargiulo S, et al. Early onset may predict G101W CDKN2A founder mutation carrier status in Ligurian melanoma patients. Melanoma Res 2004; 14:443-448.

9 Huber J, Ramos ES. The P48T germline mutation and polymorphism in the CDKN2A gene of patients with melanoma. Braz J Med Biol Res 2006; 39:237-241.

10 Della Torre G, Pasini B, Frigerio S, Donghi R, Rovini D, Delia D, et al. CDKN2A and CDK4 mutation analysis in Italian melanoma-prone families: functional characterization of a novel CDKN2A germ line mutation. $\mathrm{Br} J$ Cancer 2001; 85:836-844.

11 Fargnoli MC, Chimenti S, Keller G, Soyer HP, Dal PV, Hofler H, Peris K. CDKN2a/p16INK4a mutations and lack of p19ARF involvement in familial melanoma kindreds. J Invest Dermatol 1998; 111:1202-1206.

12 Brotherton DH, Dhanaraj V, Wick S, Brizuela L, Domaille PJ, Volyanik E, et al. Crystal structure of the complex of the cyclin D-dependent kinase Cdk6 bound to the cell-cycle inhibitor p19INK4d. Nature 1998; 395:244-250.

13 Ruas M, Peters G. The p16INK4a/CDKN2A tumor suppressor and its relatives. Biochim Biophys Acta 1998; 1378:F115-F177.

14 Soufir N, Lacapere JJ, Bertrand G, Matichard E, Meziani R, Mirebeau D, et al. Germline mutations of the INK4a-ARF gene in patients with suspected genetic predisposition to melanoma. Br J Cancer 2004; 90:503-509.

15 Borg A, Sandberg T, Nilsson K, Johannsson O, Klinker M, Masback A, et al. High frequency of multiple melanomas and breast and pancreas carcinomas in CDKN2A mutation-positive melanoma families. J Natl Cancer Inst 2000; 92:1260-1266.

16 Levanat S, Situm M, Crnic I, Marasovic D, Puizina-lvic N, Pokupcic N, et al. Alterations in CDKN2A locus as potential indicator of melanoma predisposition in relatives of non-familial melanoma cases. Croat Med J 2003; 44:418-424.

17 Platz A, Hansson J, Mansson-Brahme E, Lagerlof B, Linder S, Lundqvist E, et al. Screening of germline mutations in the CDKN2A and CDKN2B genes in Swedish families with hereditary cutaneous melanoma. J Natl Cancer Inst 1997; 89:697-702.

18 Bishop DT, Demenais F, Goldstein AM, Bergman W, Bishop JN, Bressac-de Paillerets B, et al. Geographical variation in the penetrance of CDKN2A mutations for melanoma. J Natl Cancer Inst 2002; 94:894-903.

19 Gruis NA, van der Velden PA, Sandkuijl LA, Prins DE, Weaver-Feldhaus J, Kamb A, et al. Homozygotes for CDKN2 (p16) germline mutation in Dutch familial melanoma kindreds. Nat Genet 1995; 10:351-353. 